\title{
HIGH FLUX THERMAL MANAGEMENT WITH CMOS COMPATIBLE MICROSTRUCTURED SURFACES
}

\author{
K.-H. Chu ${ }^{l *}$, R. Enright ${ }^{1,2}$, and E. N. Wang $^{l}$
}

${ }^{1}$ Department of Mechanical Engineering, Massachusetts Institute of Technology, Cambridge, Massachusetts, USA

${ }^{2}$ Stokes Institute, University of Limerick, Limerick, Ireland

\begin{abstract}
We demonstrated an enhancement in critical heat flux (CHF) of $\sim 160 \%$ on the microstructured surfaces. The fabrication process used for making the structures is CMOS-compatible and can be integrated into semiconductor processing. A simple force-balancebased model for $\mathrm{CHF}$ was developed and shows excellent agreement with the experimental observations. Based on the model predication, a potential surface design to achieve ultra high CHF is further suggested in the paper. This study shows exciting new insights into achieving high $\mathrm{CHF}$ with microstructures and provides design guidelines for new surface technologies with high heat removal capability for advanced thermal management.
\end{abstract}

\section{INTRODUCTION}

Thermal management with two-phase cooling has received significant interest for high flux applications including concentrated photovoltaics, GaN power amplifiers, and integrated circuits [1-4]. The critical heat flux (CHF) represents the operational limit in a two-phase (boiling) heat transfer system marking the point when a vapor film will begin to cover the heated surface, significantly reducing heat transfer efficiency. Therefore, methods to extend CHF have been studied extensively owing to its significant practical importance in high performance thermal management systems [5-7]. Recent efforts have focused on pushing the limits of CHF by improving surface wettability using decreased feature sizes to nanometer length scales $(\sim 100 \mathrm{~nm})$. While CHF values of $\sim 200 \mathrm{~W} / \mathrm{cm}^{2}$ with water have been reported [8-10], these nanostructures were fabricated with materials (e.g., $\mathrm{ZnO}, \mathrm{Cu}$ ) or required fabrication processes (e.g., anodic oxidation, electroless etching) that are not compatible with CMOS processing. In addition, these nanostructured surfaces were not optimized due to the limited understanding of the role of roughness-augmented wettability on CHF.

In this work, we demonstrate that with silicon microstructures alone $(\geq 5 \mu \mathrm{m})$ fabricated using standard MEMS processing, critical heat fluxes $\mathrm{q}_{\mathrm{CHF}}^{\prime \prime}>200 \mathrm{~W} / \mathrm{cm}^{2}$ are achievable. Furthermore, an analytical force-balance model was extended to explain the CHF enhancement. The excellent agreement found between the model and experimental data supports the idea that roughness-amplified capillary forces are responsible for $\mathrm{CHF}$ enhancement on structured surfaces. The work suggests that the ultra high heat removal capability $\left(>250 \mathrm{~W} / \mathrm{cm}^{2}\right)$ with structured surfaces using CMOS-compatible processing is possible.

\section{FABRICATION AND BOILING SETUP}

Microstructured surfaces with various roughness $r$, defined as the ratio of the true area in contact with the liquid to the projected area, were fabricated using MEMS processing on silicon (Fig. 1). The micropillars were patterned using projection lithography, and etched in silicon with deep reactive ion etching (DRIE). The micropillars with diameter of $5-10 \mu \mathrm{m}$, center-to-center spacings of $5-15 \mu \mathrm{m}$, and heights of $10-20 \mu \mathrm{m}$ were designed to ensure wicking behaviors [11]. Details of the pillar geometries fabricated are listed in Table I. A $300 \mathrm{~nm}$ thick thermal oxide layer was subsequently grown to enhance surface wettability. Finally, the

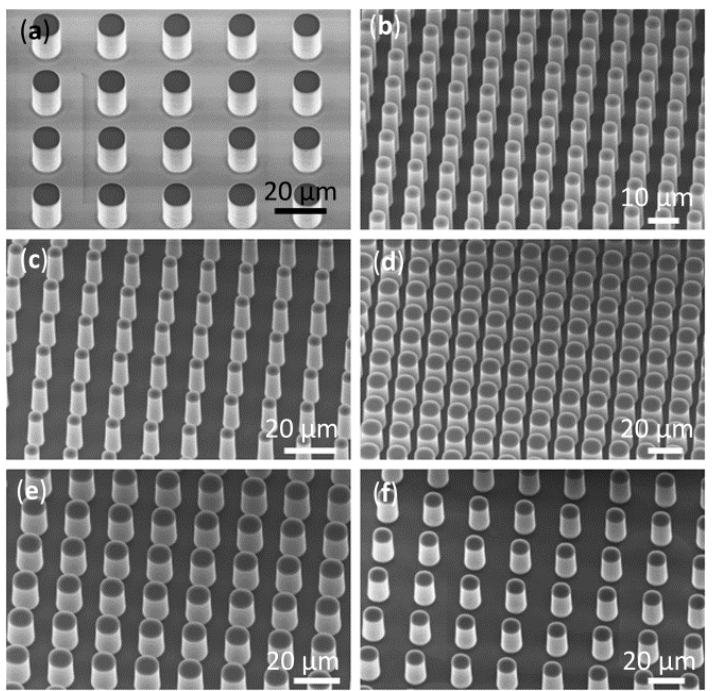

Figure 1: Scanning electron micrographs (SEMS) of the fabricated silicon microstructured surfaces. The pillars have heights of (a) $10 \mu \mathrm{m}$ and (b-f) $20 \mu \mathrm{m}$; center-to-center spacings of (a,f) $15 \mu \mathrm{m}$, $(b, d) 5 \mu \mathrm{m}$, and $(c, e) 10 \mu \mathrm{m}$; and diameters of $(b, c) 5 \mu \mathrm{m}$ and $(a$, d-f) $10 \mu \mathrm{m}$.

etched wafers were diced into samples measuring $2 \times 2 \mathrm{~cm}$, which is large enough to be considered representative of an infinite plate $[12,13]$ and of comparable size to high heat flux electronic components. Smooth oxidized samples were also prepared as benchmarks for comparison.

Fig. 2 shows the pool boiling setup which consists of an oxygen-free copper block and a tempered glass chamber fixed at both ends by Ultem mounts. Five cartridge heaters were imbedded in the copper block allowing for a maximum power of $1400 \mathrm{~W}$ $\left(350 \mathrm{~W} / \mathrm{cm}^{2}\right)$. Five in-line K- type thermocouples (KMQSS-020, OMEGA) were inserted into the center axis of the copper block with the topmost thermocouple located right beneath the sample to accurately determine the heat flux from the linear temperature gradient using Fourier's law. Note that the flux area of the copper block matched the structured sample area $(2 \times 2 \mathrm{~cm})$. A sheathed K-type thermocouple (KQSS-18U-12, OMEGA) was positioned $2 \mathrm{~cm}$ above the mounted sample to monitor the pool temperature. In the experiments, temperature was recorded with a thermocouple logger (18200-75, Cole-Parmer). To minimize losses, the chamber was wrapped in guard heaters and a layer of dense fiber glass insulation allowing the pool to be maintained at saturation temperature during the experiment.

Before experiments, the samples were bonded to the copper block using solder paste (Delta 717D, Qualitek) to ensure good attachment with minimal thermal resistance. Note that a $1 \mu \mathrm{m}$ thick copper layer was deposited on the back side of the samples to facilitate attachment to the boiling setup. For all tests, degassed, high purity water (CHROMASOLV for HPLC, Sigma-Aldrich) was used to avoid premature bubble formation and minimize surface contamination. 


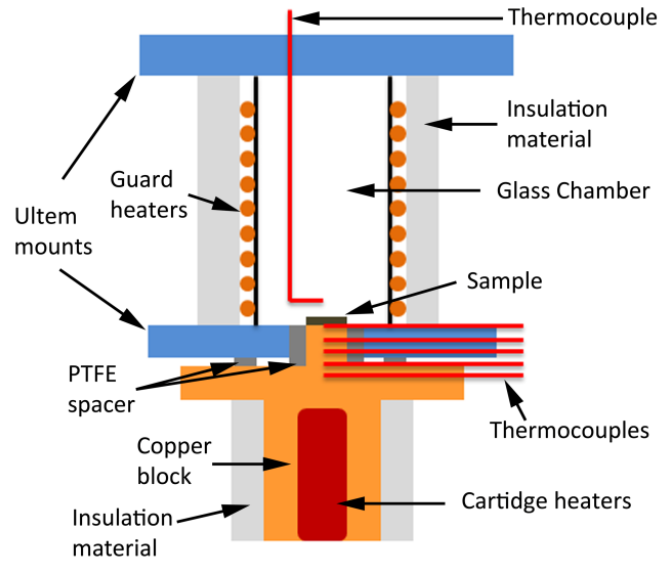

Figure. 2: Schematic of the experimental pool boiling setup with cartridge heaters in the copper block to heat the sample and inline thermocouples to accurately determine the heat flux and surface temperature. The microstructured surfaces were bonded to the copper block using solder to ensure good attachment with minimal thermal resistance.

TABLE I: Geometric parameters of the micropillar arrays. The units of height, diameter and (center-to-center) spacing are in microns. The roughness of contact line, $r$, and solid fraction, $\varphi$, are calculated by: $r=1+\pi d h(\pi / 2) /(d+s)^{2}$ and $\varphi=\left(\pi d^{2} / 4\right) /(d+s)^{2}$, respectively.

\begin{tabular}{c|c|c|c|c|c}
\hline \hline $\begin{array}{c}\text { Sample } \\
\text { No. }\end{array}$ & $\begin{array}{c}\text { Height } \\
(h)\end{array}$ & $\begin{array}{c}\text { Diameter } \\
(d)\end{array}$ & $\begin{array}{c}\text { Spacing } \\
(s)\end{array}$ & $r$ & $\varphi$ \\
\hline S1 & 10 & 10 & 15 & 1.790 & 0.126 \\
S2 & 20 & 10 & 15 & 2.579 & 0.126 \\
S3 & 20 & 5 & 10 & 3.193 & 0.087 \\
S4 & 20 & 10 & 10 & 3.467 & 0.196 \\
S5 & 20 & 10 & 5 & 5.386 & 0.349 \\
S6 & 20 & 5 & 5 & 5.935 & 0.196 \\
\hline \hline
\end{tabular}

\section{EXPERIMENTAL RESULTS}

The heat flux $q^{\prime \prime}$ as a function of wall superheat $\Delta T=T_{w}-$ $T_{\text {sat }}$, where $T_{w}$ is the heated surface temperature and $T_{\text {sat }}$ is the saturation temperature, for the smooth and microstructured $\mathrm{SiO}_{2}$ surfaces are shown in Fig. 3. The maximum uncertainty of the heat flux and temperature measurements was $\sim 5.6 \%$ and $1.8 \mathrm{~K}$, respectively. Note that in the calculation of the roughness, the scalloped features on the sidewall of micropillars were accounted for by multiplying the pillar height by a factor of $\pi / 2$ [14]. Compared with the results on the smooth surface (Sm), the boiling curves showed a significant enhancement in CHF on the structured surfaces (S1-S6). A CHF of $207.9 \pm 9.9 \mathrm{~W} / \mathrm{cm}^{2}$, which is comparable to the highest $\mathrm{CHF}$ value reported in previous studies on nanostructured surfaces [8-10, 13], was achieved with a $\Delta T=39.3 \pm 1.8 \mathrm{~K}$ on $\mathrm{S} 6$. The results demonstrate the positive correlation between the CHF and surface roughness. In addition, the two nearly identical boiling curves for the smooth surface (Sm) demonstrate the consistency and accuracy of the measurements.

\section{CHF MODEL}

To understand and predict CHF on structured surfaces where the liquid wets the surface completely, a model incorporating surface properties (i.e., surface roughness) is required. While a detailed understanding of the CHF mechanism is still lacking [15],

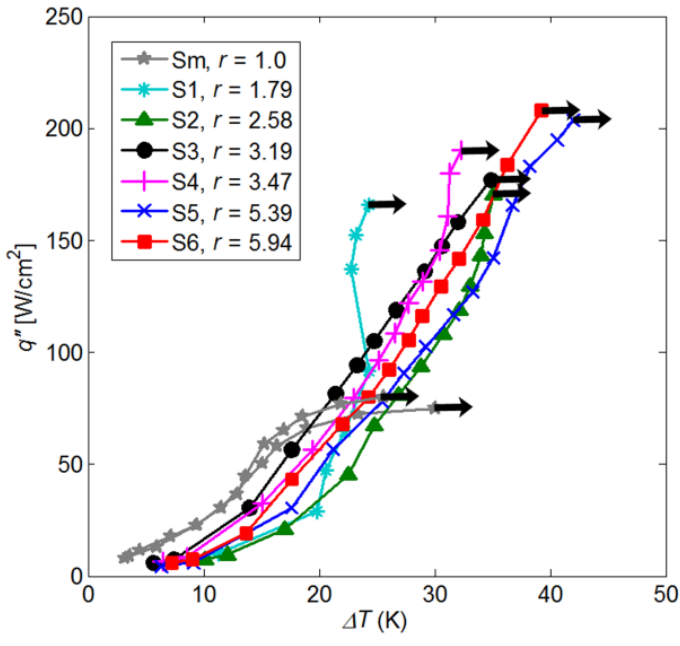

Figure 3: Boiling curves on the smooth $(r=1)$ and microstructured $(r>1)$ surfaces detailed in Table I. The arrows indicate the CHF condition. The consistency and accuracy of the measurements are demonstrated by the two nearly identical boiling curves for the smooth surface. The boiling curves show a clear trend of increasing CHF with surface roughness due to roughnessaugmented capillary forces

it is clear that surface wettability is a key factor dictating CHF [12, 15-20]. Recent works have used the capillary pumping mechanism, which assumes that there is insufficient liquid supply to balance the rate of evaporation, to predict CHF on structured surfaces [8, 16]. However, this model over-predicted $\mathrm{CHF}$ values for our microstructured surfaces $(17-120 \times$ greater than the experimental results), which suggests another mechanism dominates CHF in this case. Kandlikar presented a simplified force-based analysis for smooth surfaces assuming that there is sufficient liquid supply at CHF. Momentum, buoyancy, and surface forces at the liquid/vapor interface of an individual bubble were considered [5]. If the combination of surface and buoyancy forces compensate the momentum force during the growth phase of the bubble, the hot/dry area developed at the base of bubble during growth can rewet upon departure. Otherwise, the hot/dry area will expand irreversibly leading to the CHF condition $[15,20]$. However, in recent studies the data comparing the predictions of the Kandlikar model to the CHF behavior on structured surfaces was typically presented as a function of apparent liquid receding angle $\beta$, which leads to crowding when $\beta \rightarrow 0[9,10]$. This result is attributed to the fact that the model couples bubble geometry and the surface force through the macroscopic contact angle [5]. Thus, on superhydrophilic surfaces the model cannot account for a wide variety of structured surfaces that display no apparent contact angle, i.e., $\beta=0[8-10]$.

To address this issue, we modified the force-balance-based model to predict CHF on superhydrophilic surfaces $(\beta=0)$. In this regime, the microlayer (which includes the structures) underneath the bubble dries out such that a "Wenzel" bubble [21] is formed at CHF [15] as shown in Fig. 4. Therefore, the surface force per unit length maintaining the position of the contact line (i.e., $F_{S, 2}$ in Fig. 4) is amplified due to a longer effective contact line length, which can be estimated, assuming a bubble size larger than the underlying roughness length scale, as the unit length multiplied by the surface roughness,

$$
F_{S, 2}=\sigma_{l v} \times r \cos \theta_{r e c},
$$




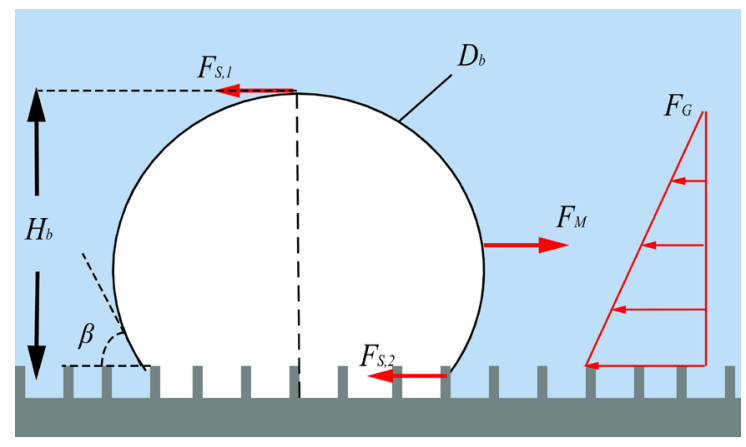

Figure 4: Schematic diagram of horizontal forces acting on the vapor bubble on a structured surface adapted from Kandlikar [5]. At CHF, the vapor film forms within microstructures beneath the bubble, i.e., "Wenzel" bubble. Here $F_{M}$ represents the force due to momentum change while $F_{G}$ represents the buoyancy force, and $F_{S, 1}$ and $F_{S, 2}$ are surface forces. $\beta$ is the apparent liquid receding angle on the structured surface, $H_{b}$ is the height of the bubble and $D_{b}$ is the diameter of the bubble

where $\sigma_{l v}$ is the liquid-vapor surface tension and $\theta_{\text {rec }}$ is the liquid receding angle on the corresponding smooth surface. CHF occurs when momentum force $F_{M}$ is greater than the sum of surface forces $F_{S, 1}, F_{S, 2}$, and buoyancy force $F_{G}$ [5]. Therefore, at CHF, the force balance in horizontal direction yields

$$
F_{M}=F_{S, 1}+F_{S, 2}+F_{G}
$$

Following the set of assumptions introduced by Kandlikar, an expression for $\mathrm{CHF}$ was obtained in the following form:

$$
q_{c}^{\prime \prime}=K \times h_{f g} \rho_{g}^{1 / 2}\left[\sigma_{l v} g\left(\rho_{l}-\rho_{g}\right)\right]^{1 / 4},
$$

where

$$
K=\left(\frac{1+\cos \beta}{16}\right)\left[\frac{2(1+\alpha)}{\pi(1+\cos \beta)}+\frac{\pi}{4}(1+\cos \beta) \cos \psi\right]^{1 / 2},
$$

$\alpha=r \cos \theta_{r e c}, h_{f g}$ is the latent heat, $\rho_{g}$ is the vapor density, and $\psi$ is the inclined angle of surface (i.e., $\psi=0$ for a horizontal upward facing surface). Note that when $\alpha<1$, Eq. 3 simplifies to Kandlikar's model. In this form, the surface force is no longer coupled with the bubble geometry such that Eq. 3 is well-defined in the complete wetting regime where $\cos \beta=1$, but $\alpha>1$.

To demonstrate the applicability of Eq. 3, the predicted CHF as a function of $\alpha$ was overlaid with data from our experiments and previous studies [8-10] in Fig. 5. Also plotted for comparison is the CHF predicted by the classical Kutateladze-Zuber (K-Z) model $[22,23]$ (hydrodynamic instability mechanism) using an empirical factor of $K=0.18$ in Eq. 3 [24]. The $r$ values for Chen et al. [8], Kim et al. [9] and Ahn et al. [10] were estimated based on the reported geometrical parameters and SEMs. These values may be inaccurate due to the fact that the surface roughness was, either, not explicitly reported or the calculation method was not detailed. In addition, contact angles reported in the literature are typically equilibrium values $\left(\theta_{e q}\right)$ measured at room temperature $T_{a}$ [8-10, 13]. Since the surface wettability is a key parameter in determining $\mathrm{CHF}$, the dependence of contact angle and surface tension on temperature should be accounted for $[5,15,19]$. Therefore, to compare the data with our CHF model, estimations for contact angles at the saturation temperature, $T_{\text {sat }}=100{ }^{\circ} \mathrm{C}$, are necessary.
Here, we estimated the variation of $\cos \theta$ with temperature from the Young-Dupré equation [25],

$$
\cos \theta(T)=W_{l s} / \sigma_{l v}(T)-1,
$$

where $\sigma_{l v}(T)$ is the temperature-dependant liquid-vapor surface tension[26] and $W_{l s}$, the work of adhesion between the liquid and solid, was estimated as

$$
W_{l s} \approx 2 \sqrt{\sigma_{s v}^{d} \sigma_{l v}^{d}(T)}
$$

where $\sigma_{s v}$ is the solid-vapor surface tension and the superscript, $d$, represents the dispersive component of surface tension. While $\sigma_{s v}^{d}$ should be only a weak function of temperature, varying by less than $1 \%$ over the investigated range from ambient to saturated temperature, the strong temperature-dependent $\sigma_{l v}^{d}$ is determined from detailed calculations [27].

In Fig. 5, the symbols represent experimental results with estimated $\theta_{\text {rec }}\left(T_{\text {sat }}\right)$ from literature [8-10] and our measurement (solid symbols) and results with $\theta_{\text {rec }}\left(T_{a}\right)$ (hollow symbols) as benchmark values. The difference in $\alpha$ between data from the literature and our estimates demonstrates the potential significance of temperature-dependant liquid contact angle on the amplified surface force (Eq. 1). The error bars for our data along the $\alpha$-axis are based on the uncertainty in the contact angle measurement at $T_{a}$, while the error bars on $\alpha$ for the literature data are due to fact that only $\theta_{e q}$ is reported rather than $\theta_{\text {rec }}$ [8-10]. For these cases, $\alpha$ was estimated using $\theta_{e q} / 2$ (i.e., average between $\theta_{e q}$ and 0 ) where the error in $\theta_{\text {rec }}$ ranged from $\theta_{e q}$ to 0 . The error bars for CHF from Ahn et al. [10] were not reported, and are therefore not shown in Fig. 5. Note that the estimated $\alpha$ values for the data of Kim et al. [9] in the complete wetting regime are shown in the inset of Fig. 5 because of the very large estimated $\alpha$ values $(\alpha \sim 55)$ due to the large reported surface roughness for their nanostructures

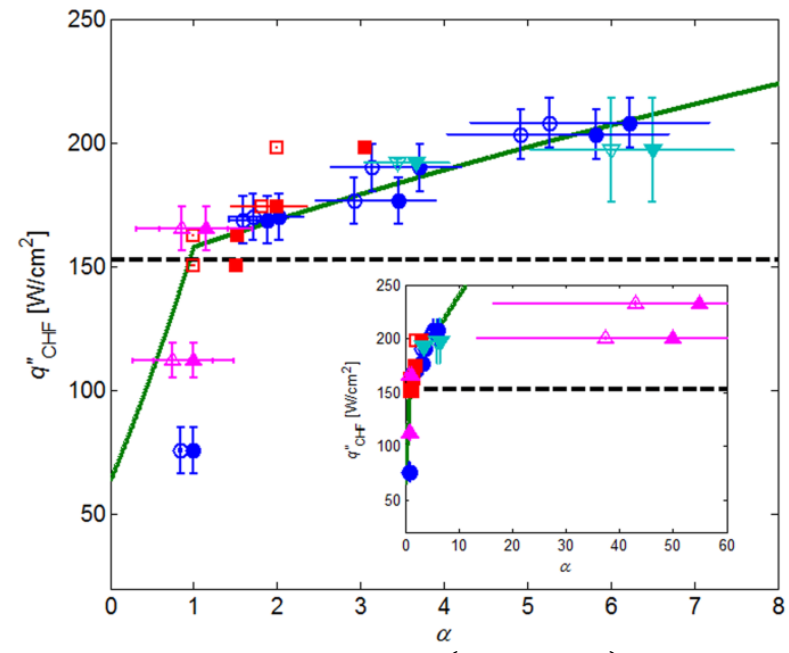

Figure 5: $C H F$ as function of $\alpha\left(=r \cos \theta_{\text {rec }}\right)$. The proposed model (solid line) is compared to the K-Z model [22, 23] with a factor of $K=0.18$ [24] (dashed line) which has been commonly accepted in the past. The symbols show the CHF data from $(\bullet)$ our

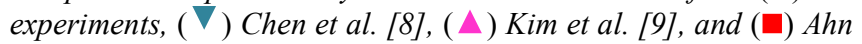
et al. [10] as a function of $\alpha$. The hollow symbols show the data from literature [8-10] while the solid symbols show the results adjusted using our estimation of $\theta_{\text {rec }}\left(T_{\text {sat }}\right)$. The inset shows data from Kim et al. [9] with surface roughness $r \sim 50-55$. 
$(r \sim 50)$ and the approximate nature of our temperature-dependent contact angle analysis. The wide error bars shown in the inset are due to the estimated error of the receding angle (ranging from $\theta_{e q}$ to 0$)$ and the large reported roughness. The result highlights the importance of properly characterizing the wetting properties.

While the value of $\alpha$ has been approximated and simplifications in the extended model exist, the good agreement between the data and model, which does not contain any fitting parameters, suggests that the key physics of the CHF mechanism on these structured surfaces are accounted for. Most importantly, the trend of a small increase in CHF with increasing surface roughness, relative to the regime where $\alpha<1$, is well-captured by the extended model. In addition, the sudden slope reduction predicted by the extended model at $\alpha=1$ can explain how the K-Z model remains well-correlated to $\mathrm{CHF}$ behavior on a range of typical engineering surfaces studied in the past where $\alpha<1.5$ (i.e., native metal oxide). Our extended model also suggests that the $\mathrm{CHF}$ of $\sim 250 \mathrm{~W} / \mathrm{cm}^{2}$ is realizable when $\alpha$ is $\sim 11-12$, which can be achieved by increasing the height of micropillar to $40-50 \mu \mathrm{m}$. However, the reduction in slope of our model for $\alpha>1$ implies that a large increase in surface roughness is required to further enhance $\mathrm{CHF}$, which offers significant fabrication challenges. One potential solution, as demonstrated by Kim et al. [9], is the use of hierarchical structures comprised of multiple roughness length scales. Following the logic suggested by Eq. 1, the effective roughness of a hierarchical surface is estimated as the product of the roughness of each length scale (i.e., $r_{e f f}=\prod_{1}^{N} r_{N}$, where $r_{N}$ is the roughness of each distinct length scale).

\section{CONCLUSION}

An enhancement in $\mathrm{CHF}$ of $\sim 160 \%$ and a $\mathrm{CHF}$ of $\sim 208 \mathrm{~W} / \mathrm{cm}^{2}$, which is comparable to the highest CHF value reported in previous studies on nanostructured surfaces, was demonstrated on the CMOS-compatible microstructured surfaces. To explain the experimental observations, we extended an analytical force balance model to predict CHF in the complete wetting regime. The model shows good agreement with the experimental observations which demonstrates the important effect of roughness-augmented wettability on CHF. The issues of contact angle variation with temperature were also addressed and should be considered in future studies given the nature of the $\mathrm{CHF}$ mechanism presented here. Furthermore, we propose using a hierarchical surface to achieve ultra high CHF based on the model predication. This study shows new insight of the role of structured surfaces in enhancing CHF and suggests opportunities to tailor advanced surface technologies using CMOS-compatible processes to achieve high heat removal for high-power electronics cooling.

\section{ACKNOWLEDGEMENT}

The authors gratefully acknowledge funding support from the Battelle Memorial Institute and the Air Force Office of Scientific Research (AFOSR). R.E. also acknowledges funding received from the Irish Research Council for Science, Engineering, and Technology, cofunded by Marie Curie Actions under FP7.

\section{REFERENCES}

[1] D. C. Price, "A review of selected thermal management solutions for military electronic systems," IEEE Trans. Compon. Packag. Technol., vol. 26, pp. 26 - 39 March 20032003.

[2] T. W. Kenny, et al., "Advanced Cooling Technologies for Microprocessorsf," Int. J. High Speed Electron. Syst, vol. 16, pp. 301313, 2006.

[3] J. R. Thome, "The New Frontier in Heat Transfer: Microscale and Nanoscale Technologies," Heat Transfer Eng., vol. 27, pp. 1-3, 2006.
[4] E. Pop, "Energy dissipation and transport in nanoscale devices," Nano Res., vol. 3, pp. 147-169, 2010.

[5] S. G. Kandlikar, "A Theoretical Model to Predict Pool Boiling CHF Incorporating Effects of Contact Angle and Orientation," J. Heat Transfer, vol. 123, pp. 1071-1079, 2001.

[6] E. Forrest, et al., "Augmentation of nucleate boiling heat transfer and critical heat flux using nanoparticle thin-film coatings," Int. J. Heat Mass Transfer, vol. 53, pp. 58-67, 2010.

[7] C. H. Li and G. P. Peterson, "Experimental study of enhanced nucleate boiling heat transfer on uniform and modulated porous structures," FHMT, vol. 1, p. 023007, 2010.

[8] R. Chen, et al., "Nanowires for Enhanced Boiling Heat Transfer," Nano Lett., vol. 9, pp. 548-553, January 16, 20092009.

[9] S. Kim, et al., "Effects of nano-fluid and surfaces with nano structure on the increase of CHF," Exp. Therm Fluid Sci., vol. 34, pp. 487-495, May 20102010.

[10] H. S. Ahn, et al., "Effect of liquid spreading due to nano/microstructures on the critical heat flux during pool boiling," Appl. Phys. Lett., vol. 98, p. 0719082011.

[11] J. Bico, et al., "Rough wetting," Europhysics Letters, vol. 55, pp. 214$220,2001$.

[12] T. G. Theofanous, et al., "The boiling crisis phenomenon Part I: nucleation and nucleate boiling heat transfer," Exp. Therm. Fluid Sci., vol. 26, pp. 775-792, 2002.

[13] M.-C. Lu, et al., "Critical heat flux of pool boiling on Si nanowire array-coated surfaces," Int. J. Heat Mass Transfer, vol. 54, p. Int. J. Heat Mass Transfer, 2011.

[14] R. Xiao, et al., "Prediction and Optimization of Liquid Propagation in Micropillar Arrays," Langmuir, vol. 26, pp. 15070-15075, 2010.

[15] C. Gerardi, et al., "Infrared thermometry study of nanofluid pool boiling phenomena," Nanoscale Res. Lett., vol. 6, p. 232, 2011.

[16] S. G. Liter and M. Kaviany, "Pool-boiling CHF enhancement by modulated porous-layer coating: theory and experiment " Int. J. Heat Mass Tran., vol. 44, pp. 4287-4311, 2001.

[17] G. P. Narayan, et al., "Mechanism of enhancement/deterioration of boiling heat transfer using stable nanoparticle suspensions over vertical tubes.," J. Appl. Phys., vol. 102, p. 074317, 2007.

[18] S. J. Kim, et al., "Surface wettability change during pool boiling of nanofluids and its effect on critical heat flux," Int. J. Heat Mass Transfer, vol. 50, pp. 4105-4116, 2007.

[19] T. G. Theofanous and T.-N. Dinh, "High heat flux boiling and burnout as microphysical phenomena: mounting evidence and opportunities," Multiphase Sci Technol., vol. 18, pp. 1-26, 2006.

[20] T. G. Theofanous, et al., "The boiling crisis phenomenon Part II: dryout dynamics and burnout," Exp. Therm. Fluid Sci., vol. 26, pp. 793-810, 2002.

[21] R. N. Wenzel, "Resistance of Solid Surfaces to Wetting by Water," Ind. Eng. Chem., vol. 28, pp. 988-994, 1936.

[22] N. Zuber, "Hydrodynamic Aspects of Boiling Heat Transfer," AEC Report AECU-4439, 1959.

[23] S. S. Kutateladze, "On the Transition to Film. Boiling under Natural Convection," Kotloturbostroenie, vol. 3, pp. 10-12, 1948.

[24] W. M. Rohsenow, et al., Handbook of heat transfer fundamentals, 2 ed. New York: McGraw-Hill, 1985.

[25] J. N. Israelachvili, Intermolecular and Surface Forces, 3 ed.: Elsevier, 2011.

[26] E. W. Lemmon, et al., "NIST Standard Reference Database 23: Reference Fluid Thermodynamic and Transport PropertiesREFPROP, Version 8.0," ed. Gaithersburg, MD: U.S. Department of Commerce, Technology Administration, National Institute of Standards and Technology, 2007.

[27] S. Takeda, et al., "Surface $\mathrm{OH}$ group governing adsorption properties of metal oxide films," Thin Solid Films, vol. 339 pp. 220-224, 1999.

\section{CONTACT}

*K.-H. Chu, tel: +1-617-324-3311; khchu@mit.edu 